\title{
Instability of Ocular Torsion During Fixation: Cyclovergence is More Stable than Cycloversion
}

\author{
L. J. VAN RIJN,* J. VAN DER STEEN, ${ }^{*}$ H. COLLEWIJN* ${ }^{*}$
}

Received 5 July 1993; in revised form 30 September 1993

\begin{abstract}
We investigated spontaneous variation of binocular torsion. Variation was expressed as SD of torsional eye positions measured over periods up to $32 \mathrm{sec}$. Subjects viewed a single dot target for periods of 32 sec. In half of the trials a large random-dot background pattern was superimposed on the dot. The movements of both eyes were measured with scleral induction coils. Spontaneous torsional movements were largely conjugate: cyclovergence was much more stable than cycloversion. This difference was not due to roll head movements. Stability of cyclovergence was improved by the background pattern. Although overall stability (SD of position) of cycloversion was unaffected by a background, the background induced or enhanced a small-amplitude torsional nystagmus in 3 out of 4 subjects. We hypothesize that the difference in stability of cycloversion vs cyclovergence reflects the greater importance of torsional retinal correspondence, compared to absolute torsional position. In two subjects we found evidence for the existence of cyclophoria, manifested by systematic shifts in cyclovergence caused by the appearance and disappearance of the background.
\end{abstract}

Cyclovergence Cyclodisparity Cyclophoria Human Search coil

\section{INTRODUCTION}

For unblurred visual perception, retinal images need to be, within certain margins, corresponding and stable on the two retinas. Correspondence prevents the perception of double images and allows full usage of stereopsis. Stability is needed because high retinal image speeds lead to motion-blur. The quality of image stability and correspondence and their effects on perception have been well evaluated for eye movements in horizontal and vertical directions (Westheimer \& McKee, 1975; Skavenski, Hansen, Steinman \& Winterson, 1979; Steinman \& Collewijn, 1980; Steinman, Levinson, Collewijn \& Van der Steen, 1985; Erkelens \& Collewijn, 1985; Steinman, 1986; Ferman, Collewijn, Jansen \& Van den Berg, 1987a).

The effects on perception of eye movements about the torsional axis (the line of sight, see Methods) have been less well investigated. One may expect that the effect of torsional instability is less pronounced because it induces retinal image motion predominantly in the periphery of the visual field. It has indeed been found that the stability of eye torsion is much less than of horizontal and vertical eye position. Ferman et al. (1987a) reported SD values of about $0.27 \mathrm{deg}$ for torsion, within periods of fixation, compared to SD values of 6.7 and 8 min arc

\footnotetext{
*Department of Physiology I, Faculty of Medicine, Erasmus University Rotterdam, P.O. Box 1738, 3000 DR Rotterdam, The Netherlands.

$\nmid$ To whom all correspondence should be addressed.
}

for horizontal and vertical positions. Approximately similar values were reported by Ott, Seidman and Leigh (1992).

A number of significant aspects is not covered by these previous studies of torsion stability. Firstly, they dealt with monocular torsion. Therefore, they addressed monocular torsional retinal image slip, not torsional retinal correspondence. Secondly, none of those studies contains an evaluation of the role of trial length on the variability of torsion. It has been noticed that drift, i.e. prolonged motion in one direction, is a major constituent of torsional variability (Ferman et al., 1987a). Therefore, variability is likely to critically depend on the length of the sample that is considered.

A first analysis of cyclovergence variability was recently published by Enright (1990), who found that, within periods of fixation, variability of cyclovergence was much smaller than variability of monocular-torsion (SD about $4 \mathrm{~min}$ arc for cyclovergence and $17 \mathrm{~min}$ arc for monocular torsion). Between fixations, cyclovergence variability amounted to $15 \mathrm{~min}$ arc (SD). Due to limitations of Enright's measurement technique, temporal resolution was low.

A third issue that was not evaluated in previous papers is the role of visual feedback in ocular stabilisation. Several reports indicate that both cycloversion and cyclovergence can be elicited by adequate visual stimuli (Crone \& Everhard-Halm, 1975; Kertesz, 1983; Howard \& Zacher, 1991; Van Rijn, Van der Steen \& Collewijn, $1992,1994)$. Therefore, one would expect stability to be enhanced when such stimuli are present. 
The purpose of the present study was to evaluate the stability of cyclovergence and cycloversion and to compare it to the stability of horizontal and vertical vergence and version. We recorded eye movements with scleral coils, which offer excellent temporal and spatial resolution and we were therefore able to incorporate the element of trial length into the analysis. We also studied the possible role of visual feedback, by using a single dot target with and without a large, structured background.

We found that cyclovergence was more stable than cycloversion and that cyclovergence stability was enhanced by visual feedback. An important side conclusion is that coil slippage was minimal within, but not between, trials. Some preliminary results of these experiments have been presented in abstract form (Van Rijn \& Van der Steen, 1992).

\section{METHODS}

\section{Subjects}

Four human subjects ( 3 males, 1 female, age range 26-57) served in this experiment, after giving informed consent. Three of them were myopic (about -2 to $-3 \mathrm{D}$ ); one was emmetropic. All subjects had a visual acuity of at least 20/20 in each eye (measured with their own spectacle correction) and normal binocular vision. Stereoacuities were not worse than $60 \mathrm{sec}$ of arc in the TNO test for stereopsis (Medical Workshop, Groningen, The Netherlands). The myopic subjects wore their spectacles during the experiment. All subjects were experienced in wearing scleral coils.

\section{Recording of eye positions and data analysis}

Eye rotations were measured with scleral induction coils of the combination type, suitable for measurements about three axes (Robinson, 1963; Ferman et al., 1987a; manufactured by Skalar, Delft, The Netherlands). Angular positions of the coils were measured by a phase-lock technique (Robinson, 1963). The eye position signals were low-pass filtered at $62.5 \mathrm{~Hz}$, digitised at $125 \mathrm{~Hz}$ and stored on disk by a minicomputer (DEC PDP 11/73) for off-line analysis.

Prior to each experiment, gains of all channels were calibrated and offsets were zeroed. During this calibration the scleral coils were mounted in a straightahead position on a protractor device and placed near the centre of the magnetic field, similar to the position of the eyes during the experiment. The average offset values from each first trial without background in a session were used to correct all data for coil misalignment. This correction was done using a matrix transformation described by Ferman et al. (1987a). [We neglected the small adduction of each visual axis (about $1.3 \mathrm{deg}$ ) due to the finite distance $(145 \mathrm{~cm})$ of the target.] In this way we obtained veridical eye positions relative to an earth-fixed co-ordinate system that were free of cross-coupling artifacts due to coil misalignments. (Note that, as a consequence of this procedure, mean torsion was by definition zero in the first measurement without background.)

All eye rotations were expressed in Fick coordinates (see e.g. Carpenter, 1988). This implies that torsion was expressed as rotation about the line of sight. This was adequate for this experiment, because eye torsion expressed in this way is directly related to retinal image rotation. Alternatively, one may express torsion as rotation about a head-fixed antero-posterior axis (Haustein, 1989; Tweed, Cadera \& Vilis, 1990).

The noise levels of the apparatus (measured as standard deviations, SD, of the signals with the coil on the stationary protractor device) were about $0.005 \mathrm{deg}$ in horizontal and vertical directions and $0.01 \mathrm{deg}$ in torsional direction. As the SD of torsional position with the coil mounted on the eye was in most cases at least $0.04 \mathrm{deg}$, these values were affected by apparatus noise by only about $7 \%$ (comparison of variances, see e.g. Glanz, 1987). Therefore we did not correct our data for this apparatus noise.

\section{Protocol and visual stimuli}

Subjects were seated with their eyes near the centre of the magnetic field of eye-position measurement system. Their heads were supported by chin and forehead rests. At $145 \mathrm{~cm}$ distance in front of the subject a single red dot $(0.24 \mathrm{deg}$ of visual angle in diameter, luminance about $15 \mathrm{~cd} / \mathrm{m}^{2}$ ) was backprojected on a tangent translucent screen, in a straight-ahead position. Each experiment consisted of 20 trials. Subjects were instructed to fixate the dot continuously during each trial, lasting $32 \mathrm{sec}$, without blinking. Successive trials were separated by a pause of approximately $15 \mathrm{sec}$, timed by a metronome. The subject was instructed to blink several (typically about 20) times during the first $10 \mathrm{sec}$ of this pause and to abstain from blinking during the last $5 \mathrm{sec}$. At the end of the pause, the subject started the next trial. The rationale behind this instruction was to somehow "reset" torsion and thus obtain a realistic value for inter-trial variability. As later analysis suggested that, with this procedure, inter-trial variability was largely determined by coil-slippage, values for inter-trial variability will not be reported as such. During half of the trials, a square background pattern (width $\times$ height: $55 \times 52$ deg of visual angle) was superimposed upon the dot. This pattern consisted of squares measuring $0.6 \mathrm{deg}$ of visual angle. The colour of each square (either black or white) was randomised (Julesz, 1965). The brightness of the background was low (about $2 \mathrm{~cd} / \mathrm{m}^{2}$ ) so as to leave the dot clearly visible. Trials with and without the background pattern were alternated ("background" and "dark"). Sessions started with the background on in subjects 1 and 2, and with the dot only in subjects 3 and 4 . Throughout the experiment the room was thoroughly darkened. Therefore, in the absence of the background, there were no visual cues that could provide references for eye torsion. 


\section{Data analysis and statistical testing}

Prior to analysis, all traces were inspected for the occurrence of blinks. Only 4 trials ( 2 in each of subjects 2 and 3 ) needed to be excluded on this ground from further analysis.

After correction for coil-misalignment (see above), vergence and version of all movement directions (i.e. horizontal, vertical and torsional) were calculated for each data sample. Vergence was defined as left eye position minus right eye position (e.g. cyclovergence $=$ left eye torsion - right eye torsion) and version was the average of the positions of the left and right eyes (hence: cycloversion $=[$ left eye torsion + right eye torsion]/2).

Mean and SD were calculated over all version and vergence values during a $32 \mathrm{sec}$ trial. These $S D$ values $\left(\mathrm{SD}_{32}\right)$ were taken as a measure for intra-trial variability. Data shown in Figs 2 and 5 and Table 1 are averages of $\mathrm{SD}_{32}$ values.

Trend was calculated in each trial as the slope of the linear regression line through all data samples in that particular trial. For torsion, calculation of $\mathrm{SD}_{32}$ values was repeated after removal of trends (Table 1).

$\mathrm{SD}_{32}$ values were compared in an analysis of variance (ANOVA; factors: subject and background) and in paired $t$-tests (cyclovergence vs cycloversion). In order to obtain data with a (pseudo)-normal distribution, all SD values were log-transformed prior to statistical analysis.
All reported $P$-values were calculated assuming twosided alternative hypotheses.

For torsion (cyclovergence and cycloversion) we also calculated the cumulative SD as a function of elapsed time $t\left(\mathrm{SD}_{t}\right)$ for each trial. The $\mathrm{SD}_{t}$ was calculated over all samples between time 0 and time $t$ within a trial. The data shown in Fig. 3 are SD, values, averaged across trials.

\section{RESULTS}

\section{Intra-trial stability}

In all four subjects spontaneous, torsional movements were largely conjugate. Figure 1 shows typical recordings for each subject, with and without background. The traces of torsion of the left and right eyes are largely similar. As a result, the trace of cycloversion largely corresponds to those of the separate eyes, while the trace of cyclovergence is much more stable. In agreement with this, we found that the $\mathrm{SD}_{32}$ values for cycloversion were much larger than those of cyclovergence (paired $t$-test: $P<0.001$ ). This is shown in Fig. 2, which shows averages of $\mathrm{SD}_{32}$ values for each movement direction and subject.

For comparison, Fig. 2 also shows $\mathrm{SD}_{32}$ values for horizontal and vertical vergence and version eye movements. These values were much lower than those for torsion but also for horizontal and vertical movements,

\section{A: dark}

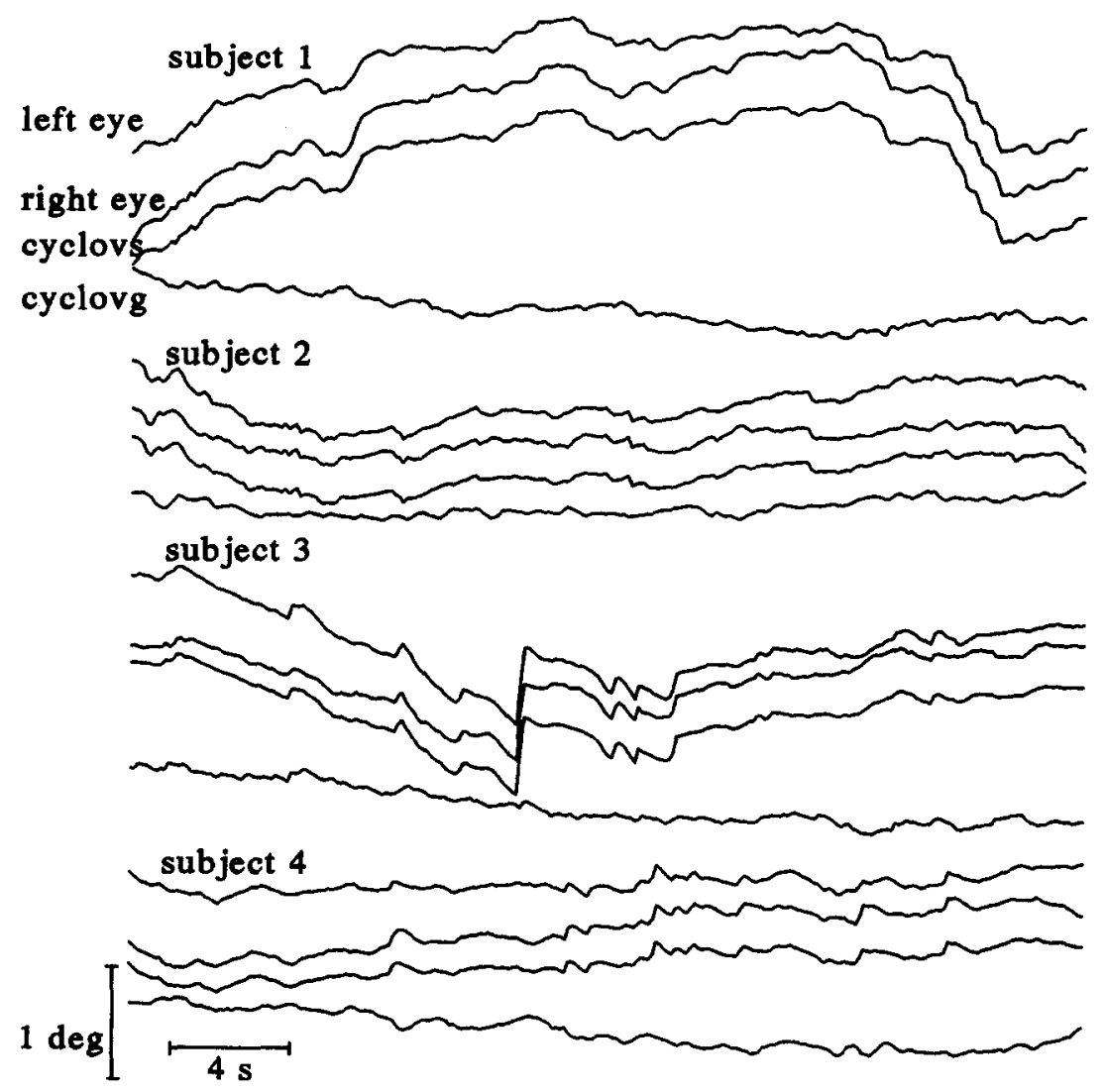

FIGURE 1(A). Caption overleaf. 


\section{B: background}
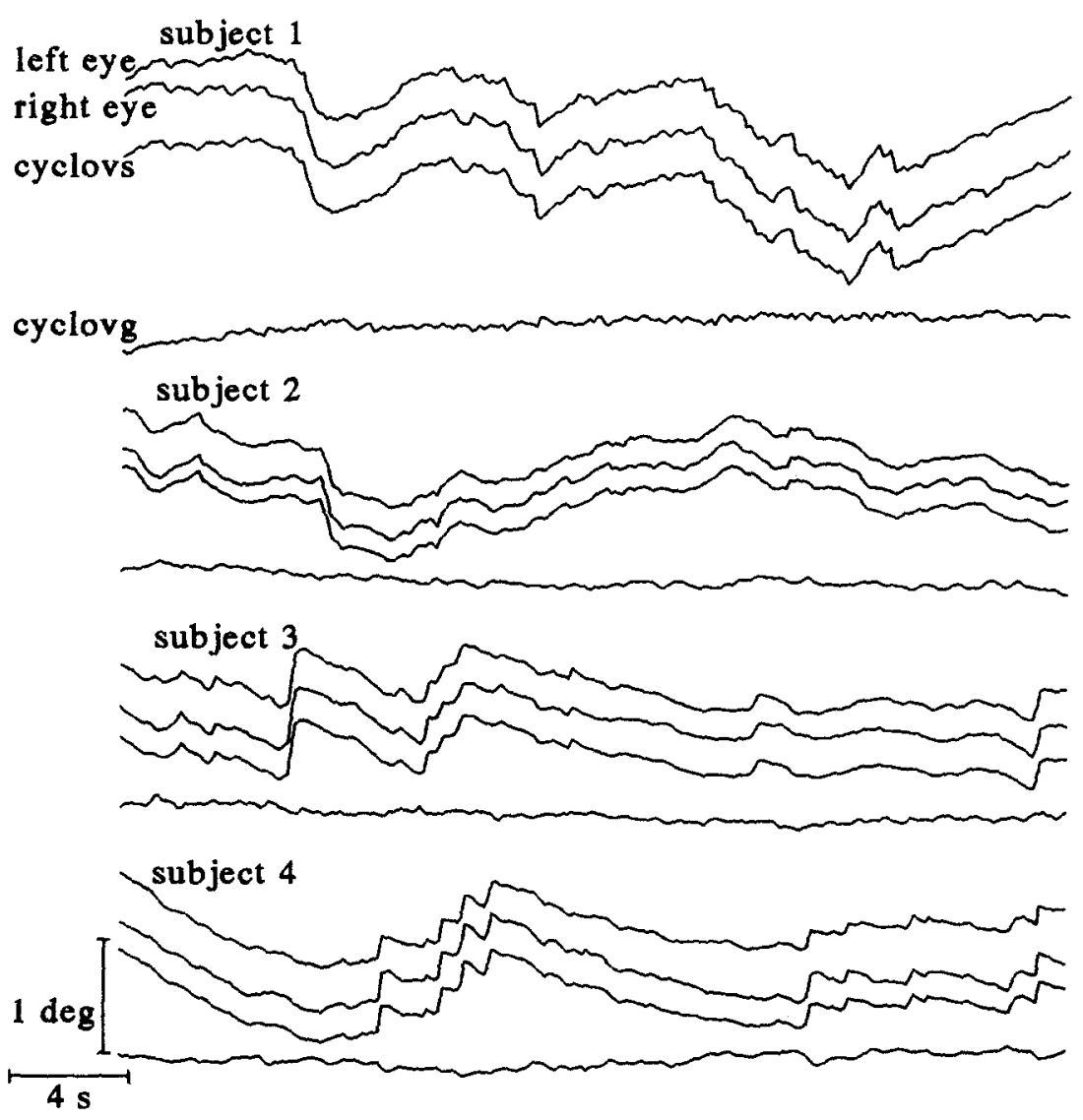

FIGURE 1. Typical recordings of torsion (whole trials, lasting $32 \mathrm{sec}$ ) of each of the 4 subjects. For each subject 4 traces are shown: left eye torsion, right eye torsion, cycloversion (cyclovs) and cyclovergence (cyclovg), without (A) and with a random-dot background (B). Traces of the individual eyes correspond closely to each other and to the traces of cycloversion. As a result, the cyclovergence traces hardly show any variation.

vergence was more stable than version (both $P$ values $<0.001)$

\section{Effect of background}

In all subjects, cyclovergence stability was markedly enhanced by the background (ANOVA: $P<0.001$ ). For cycloversion, such an effect was absent (ANOVA: $P=0.878$ ). The effect of the background on torsion stability is demonstrated in Table 1 (panel A: overall variability), which shows variability values of cycloversion and cyclovergence, separated according to background condition. There were also effect of the background on stability about the other directions of motion (not illustrated). Horizontal vergence was also more stable in the presence of the background $(P=0.002)$, without any effect on horizontal version $(P=0.927)$. The presence of a background did not significantly affect vertical vergence $(P=0.136)$ but vertical version was significantly less stable with the background ( $P=0.034$ ). This was due to the induction, by the background, of a small vertical nystagmus, consisting of slow and fast phases, in subjects 2 and 3 . In subjects 1, 3 and 4 the background also induced a slight torsional (cycloversional) nystagmus (Fig. 1). This did not significantly affect $\mathbf{S D}_{32}$ values, because the amplitude of the nystagmus was small, compared to the overall variability.

\section{Trends in cycloversion and cyclovergence}

Trends in cycloversion and cyclovergence, calculated over the entire $32 \mathrm{sec}$ trial length were very small in subjects 2 and 3 and more substantial only in subjects 1 and 4 . In subject 1 , trends in cyclovergence were oppositely directed in trials with background as compared to those without background (dark: $0.007 \pm 0.014 \mathrm{deg} / \mathrm{sec}$; background: $-0.020 \pm 0.016 \mathrm{deg} / \mathrm{sec})$. These differences in trend values between subsequent trials were systematic. As will be discussed later, this kind of trend may be interpreted as the slow establishment (dark) and the slow correction (background) of a cyclophoria (the major fraction of this cyclophoria was established or corrected between trials; see Fig. 4 and below). In subject 4, trends were always in the direction of ex-cyclovergence, irrespective of background condition $(-0.008 \pm 0.008 \mathrm{deg} /$ sec and $-0.008 \pm 0.006 \mathrm{deg} / \mathrm{sec}$ for dark and background, respectively). As will be discussed later, such a type of trend may be related to coil slippage, induced by inter-trial blinking. In subjects 2 and 3 , trends were 


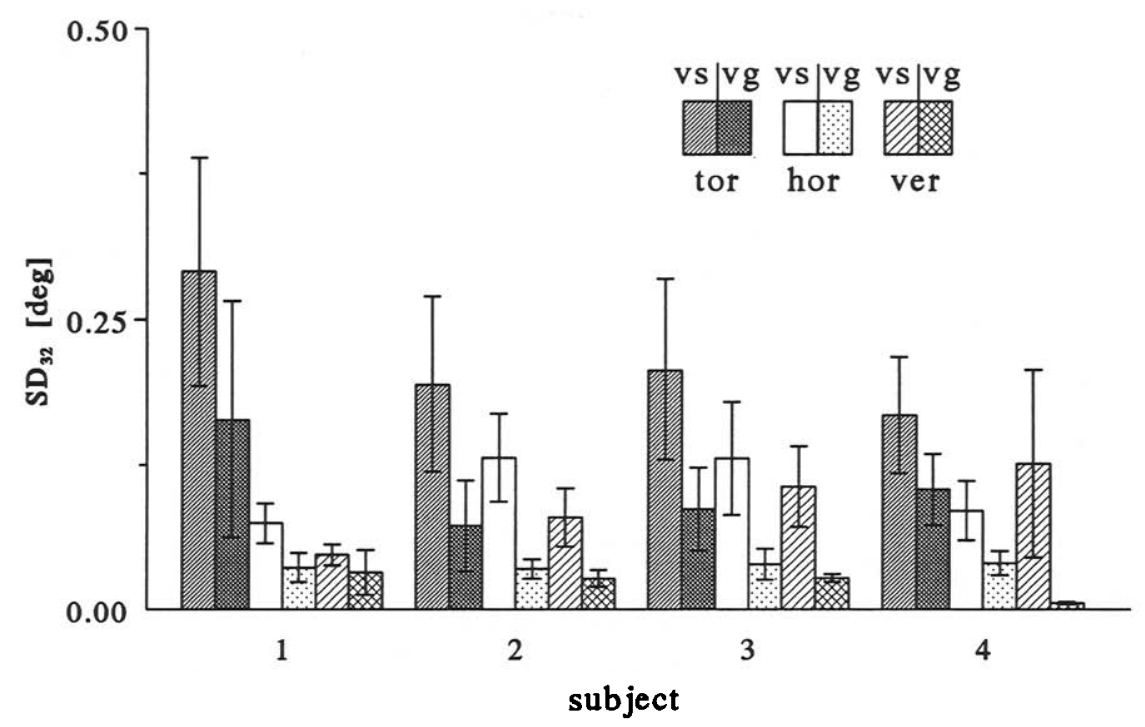

FIGURE 2. Variability of version (vs) and vergence (vg) in all three movement directions (horizontal, vertical and torsion), expressed as $\mathrm{SD}_{32}$, i.e. as the $\mathrm{SD}$ of all data samples within a $32 \mathrm{sec}$ trial. Values are expressed as the mean \pm SD of the $\mathrm{SD}_{32}$-values of 18 trials in subjects 2 and 3 and of 20 trials in subjects 1 and 4 . Stability of vergence was better than that of version in all movement directions. Horizontal and vertical version and vergence were more stable than torsional vergence and version.

smaller (overall 0.0007 and $-0.003 \mathrm{deg} / \mathrm{sec}$, respectively) and not systematic in direction or in relation to background condition.

After removal of the trends, the differences between cycloversion and cyclovergence stability, as well as the effect of the background on cyclovergence stability, were still present. This is demonstrated in Table 1, panel B, which shows variability values of cycloversion and cyclovergence after trend removal.

\section{Effects of trial length}

Figure 3 shows the cumulative $\mathrm{SD}\left(\mathrm{SD}_{t}\right.$, the $\mathrm{SD}$ as function of elapsed time within trials). Cyclovergence variability in subjects 2 and 3 reached a constant level after approximately $10 \mathrm{sec}$. In contrast, in subjects 1 and 4, cyclovergence variability continued to rise until approximately $20 \mathrm{sec}$. This continuous rise corresponds to the larger "trend" component of cyclovergence variability that was present in subjects 1 and 4 (see Table 1).

TABLE 1. Variability of cycloversion and cyclovergence, expressed as $\mathbf{S D}_{32}$, separated according to background condition. Cyclovergence was more stable with than without the background; for cycloversion there was no difference. Panel A: overall variability; Panel B: same after removal of within-trail trends. Cycloversion-cyclovergence differences and differences between background conditions were similar to those found before trend removal. All $\mathrm{SD}_{32}$ values are expressed as mean \pm SD of 9 trials in subjects 2 and 3 and of 10 trials in subjects 1 and 4

\begin{tabular}{lllll}
\hline & \multicolumn{3}{c}{ (A) Overall variability } \\
Subject & Cycloversion & Cyclovergence & Cycloversion & Cyclovergence \\
\hline 1 & $0.292 \pm 0.103$ & $0.247 \pm 0.0079$ & $0.290 \pm 0.097$ & $0.081 \pm 0.019$ \\
2 & $0.211 \pm 0.090$ & $0.098 \pm 0.042$ & $0.178 \pm 0.056$ & $0.047 \pm 0.009$ \\
3 & $0.216 \pm 0.075$ & $0.120 \pm 0.027$ & $0.200 \pm 0.083$ & $0.061 \pm 0.014$ \\
4 & $0.148 \pm 0.039$ & $0.115 \pm 0.032$ & $0.188 \pm 0.055$ & $0.093 \pm 0.026$ \\
Mean & $0.217 \pm 0.059$ & $0.145 \pm 0.069$ & $0.214 \pm 0.051$ & $0.071 \pm 0.020$ \\
\hline
\end{tabular}

(B) Variability after trend removal

$$
\text { Dark Background }
$$

\begin{tabular}{lcccc} 
Subject & Cycloversion & Cyclovergence & Cycloversion & Cyclovergence \\
\hline 1 & $0.259 \pm 0.084$ & $0.105 \pm 0.046$ & $0.187 \pm 0.051$ & $0.042 \pm 0.006$ \\
2 & $0.200 \pm 0.089$ & $0.081 \pm 0.021$ & $0.158 \pm 0.044$ & $0.037 \pm 0.006$ \\
3 & $0.177 \pm 0.051$ & $0.091 \pm 0.021$ & $0.172 \pm 0.080$ & $0.053 \pm 0.015$ \\
4 & $0.113 \pm 0.038$ & $0.060 \pm 0.011$ & $0.160 \pm 0.039$ & $0.044 \pm 0.009$ \\
Mean & $0.187 \pm 0.060$ & $0.084 \pm 0.019$ & $0.169 \pm 0.013$ & $0.044 \pm 0.0007$ \\
\hline
\end{tabular}



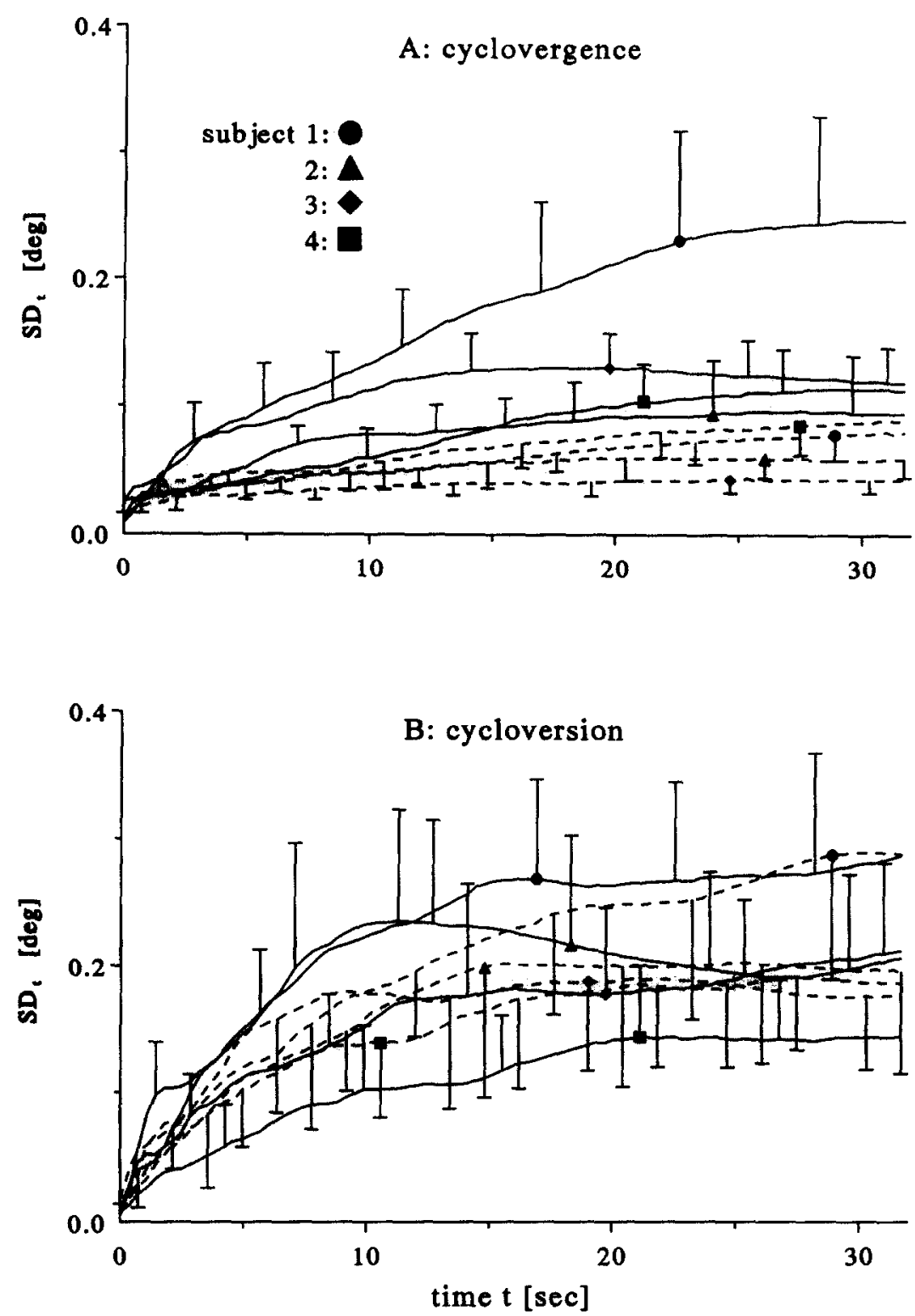

FIGURE 3. Cumulative SD within trials separated according to background condition (continuous lines, Dark; dashed lines, Background). The $\mathrm{SD}_{1}$ represents the SD, calculated over all samples between time 0 and time $t$. (A) Cyclovergence variability, which was stable after about $10 \mathrm{sec}$ in subjects 2 and 3. In subjects 1 and 4 there was a continuous rise until about 20-25 sec. Cycloversion variability, shown in (B), reached a plateau after about $15 \mathrm{sec}$ in all subjects. Values are expressed as mean \pm SD of 9 trials in subjects 2 and 3 and of 10 trials in subjects 1 and 4 . In the figure, error bars are shown for every 96th data point only.

Cycloversion variability reached constant levels after 15 to $20 \mathrm{sec}$, irrespective of subject or background condition.

Figure 3 shows once more that cyclovergence was more stable than cycloversion and that cyclovergence stability was enhanced by the background. Notice that the $\mathrm{SD}_{32}$ values reported in Fig. 2 and Table 1 represent the end-points of curves as shown in Fig. 3.

\section{Inter-fixation stability}

Figure 4 displays, for each subject, the mean cyclovergence and cycloversion angles during successive trials, as function of trial number. The cyclovergence graphs for subject 1 , and to a lesser extent subject 2 , display a typical saw-tooth pattern in which cyclovergence alternates systematically between subsequent trials. This reflects the fact that in these two subjects cyclovergence angles with the background were systematically different from those without the background. This is a clear indication of cyclophoria. In subject 1 , the background induced an in-cyclovergence; in subject 2 an ex-cyclovergence. In addition, in subject 1 , who showed the largest cyclophoria (about $2 \mathrm{deg}$ ), intra-fixational trends were towards incyclovergence with the background and towards excyclovergence without the background. Hence, the establishment and correction of cyclophoria were apparently not completed in the interval between trials. Graphs for cycloversion did not show this background dependent variation in these subjects.

Apart from these systematic changes in cyclovergence elicited by the visual background, all subjects showed, over the course of a whole session, a considerable (several degrees) shift of mean cyclovergence, always in 
the direction of in-cyclovergence. In our view this "long-term" change reflects the effects of coil slippage (see Discussion). Inspection of the traces of separate eyes (not shown) revealed that in subject 4 the in-torsional trend occurred mainly in the left eye; in subject 2 in the right eye and in subjects 1 and 3 in both eyes. This is reflected in Fig. 4 in long term cyclovergence changes in all subjects whereas cycloversion only changes in subject 4 and, to a lesser extent, 2 . In subjects 1,2 and 3 there was no relation between these long-term cyclovergence changes and either the direction or magnitude of intra-trial trends. In subject 4 the intra-trial cyclovergence drift was opposite to the "long-term" change (see discussion on coil slippage).

\section{Eye vs head stability}

Differences in stability between cycloversion and cyclovergence could, in principle, be caused by roll head movements. The torsional VOR has a low gain (on the order of 0.7 or less: Collewijn, Van der Steen, Ferman \& Jansen, 1985; Seidman \& Leigh, 1989). Therefore, head movements about an antero-posterior axis are compensated only partially by opposite torsional eye movements. In order to exclude roll head movements as a possible source of the cyclovergence-cycloversion stability differences, we repeated the experiment in one subject (subject 1) with one of the coils positioned on the left eye and the other coil mounted on the forehead. Except for this change in position of one coil, the
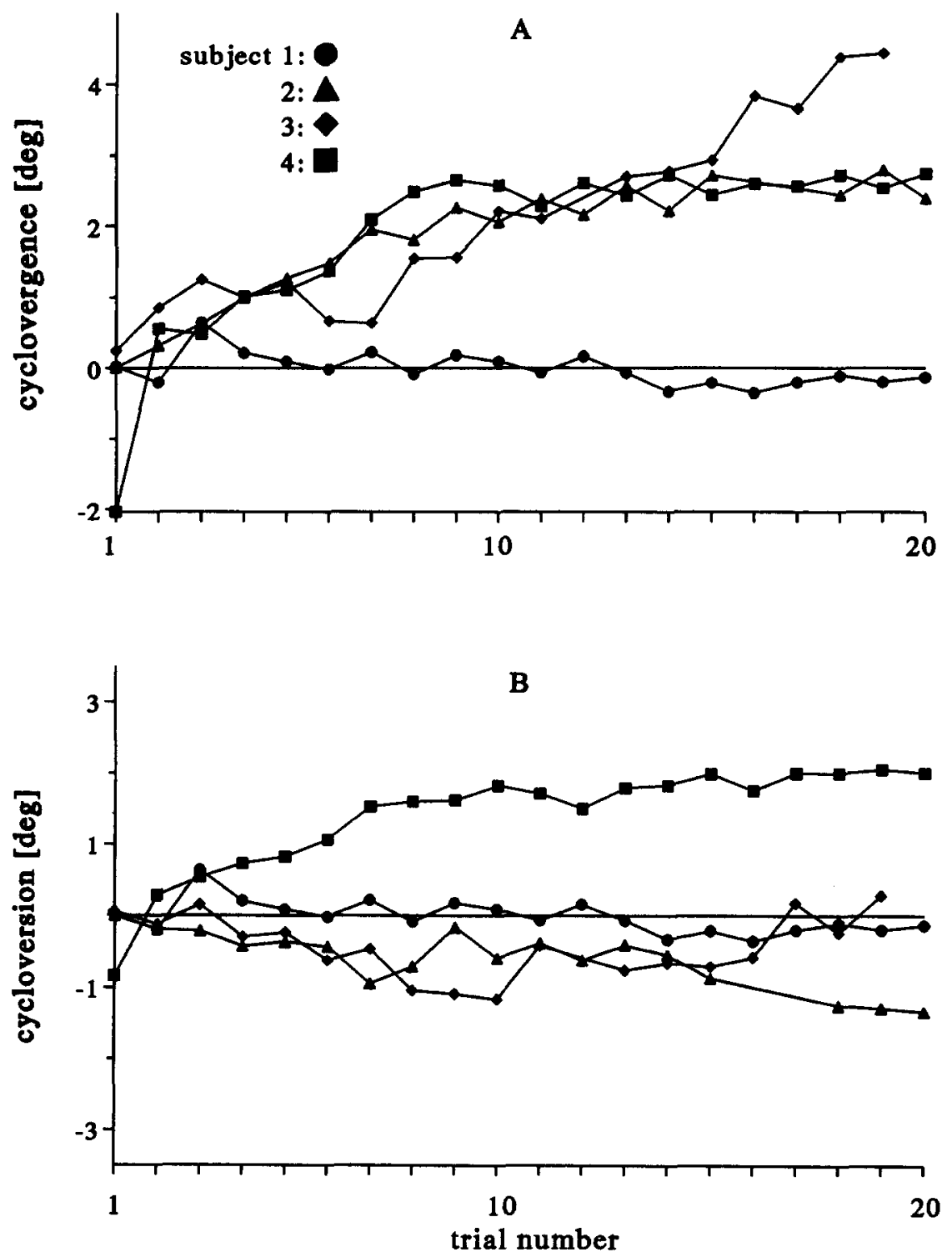

FIGURE 4. Mean cyclovergence and cycloversion during a trial, as a function of successive trial number, indicating "long-term" changes. Trials with the background are even-numbered in subjects 1 and 2 and odd-numbered in subjects 3 and 4. (A) Cyclovergence. In all subjects, over the total duration of the session there was a trend towards in-cyclovergence (positive cyclovergence values). In subjects 1 and 2 , values in darkness were systematically different from those with the background (saw-tooth pattern), indicating cyclophoria. (B) Cycloversion, which is positive for clockwise rotations. Long-term trends in cycloversion were generally less than those in cyclovergence. 


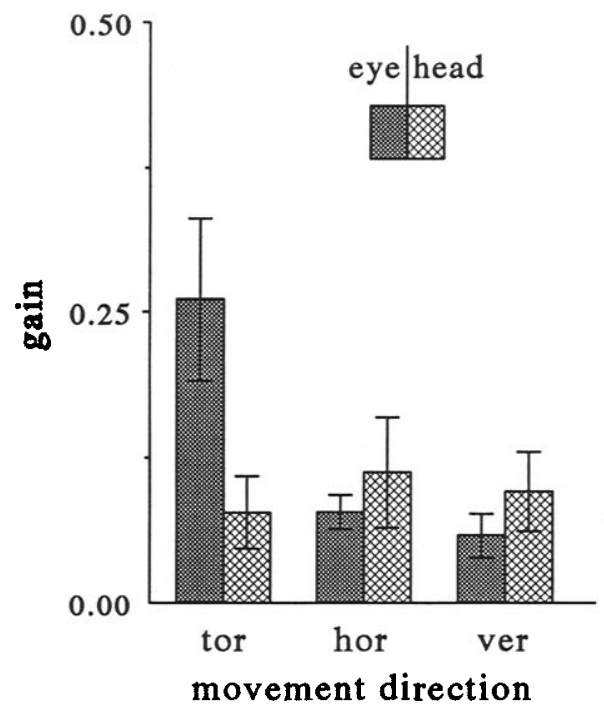

FIGURE 5. Variability of the left eye vs variability of the head, in subject 1 , for the three axes of motion. SD $_{32}$ values are calculated as the SD of all samples within a $32 \mathrm{sec}$ trial and are expressed as mean \pm SD of 20 trials. Torsional stability of the head was much better than that of the eye. In contrast, in horizontal and vertical directions eye stability was better than head stability.

protocol was identical to that of the first experiment. Results are shown in Fig. 5. Torsional stability values of the left eye corresponded to those found in the first experiment in this subject. As was pointed out above, this monocular torsional stability was similar to the stability of cycloversion (Fig. 2). Stability of the head for roll movements was far better (paired $t$-test: $P<0.001$ ). In fact, roll head movements could account for only about $5 \%$ of left eye torsion variability (comparison of variances) and could therefore not explain the cyclovergence-cycloversion differences that we found. In contrast to torsion, for horizontal and vertical movements, eye stability was significantly better than head stability (paired $t$-tests: $P=0.013$ and $P<0.001$ for horizontal and vertical movements, respectively).

\section{DISCUSSION}

In the present experiment we investigated to which extent spontaneous torsional eye movements are conjugate, i.e. if there is a difference between cycloversion and cyclovergence (within trial) variability. Also, we looked at the effect of a structured background on this variability, as well as at the effect of trial length. Finally we considered between-trial variability, which led to some conclusions concerning the existence of cyclophoria and concerning coil-on-eye stability.

\section{Cyclovergence vs cycloversion variability}

Previously, only monocular torsional variability has been analysed with the scleral coil technique. Ferman et al. (1987a) and Ott et al. (1992) found that torsion was much less stable than horizontal and vertical eye position. Although Ott et al. measured the stability in three dimensions (i.e. including torsion) of both eyes simultaneously, they did not analyse or describe vergence- version differences. Cyclovergence stability has been previously analysed only with photographic measurement techniques, which offer poor temporal resolution (Enright, 1990; Diamond, Markham \& Money, 1990).

The results from our experiment confirm the finding of these previous investigators that torsion is less stable than horizontal and vertical eye position. In addition, we find that, at least within periods of fixation, torsion is largely conjugate. As a result, cycloversion displays much more variation than cyclovergence. This implies that these spontaneous torsional eye movements do not originate from random variation in each eye but have their source in a control mechanism that is common to both eyes. We found a similar version-vergence stability difference for horizontal and vertical positions. This may be the result of voluntary versional movements in the plane of fixation. For this reason it is less surprising than the difference for torsion since, without special training (Balliet \& Nakayama, 1978) torsional movements cannot be made at will.

\section{Effect of background}

Another finding from the present experiment is that the stability of cyclovergence is much enhanced by a structured visual background. This implies that visual feedback plays an important role in maintaining cyclovergence stability. The lack of improvement by a structured background of stability of cycloversion does not imply that cycloversion is altogether unaffected by visual stimuli. In previous work (Van Rijn et al., 1992, 1994) we demonstrated that gains of cycloversion movements, induced by sinusoidal oscillation about the line of sight of similar stimulus configurations as used in the present experiment, are as high as those of cyclovergence. From these observations we may conclude that dynamic cycloversion responses are superimposed on spontaneous variation. This is in agreement with the considerable drift and variable phase values that we found for dynamic cycloversion, despite good responses (Van Rijn et al., 1992, 1994). Addition of spontaneous variation and dynamic responses is likely to occur in cyclovergence as well. A main difference between the two systems would then be that visual control of cyclovergence contains a marked static component, which maintains correspondence, while visual control of cycloversion is limited to a (modest) dynamic response to changes in orientation. This difference may be explained by the nature of the visual references that are available to the two systems. Cyclovergence is controlled by cyclodisparity, and optimal correspondence will be achieved by negative feedback control that minimises any cyclodisparity by suitable cyclovergence. Thus, the set-point for cyclovergence is zero cyclodisparity, which is an unambiguous, internal reference, based on the comparison of the two retinal images. A similar set-point for the visual control of cycloversion would require both an absolute estimate of orientation of contours on the retina and knowledge about the objective orientation of the same contours in the world. Given the variety of orientations in the world and the rules of perspective and 
optical projection, such an estimate is unlikely to be very accurate. Curiously, our results even suggest that a large-field, structured stimulus may destabilise version: the background induced or enhanced the manifestation of a small, but distinct vertical nystagmus in 2 out of our 4 subjects, and a cycloversion nystagmus in 3 subjects. A similar result was reported for monocular torsion by Ferman, Collewijn and Van den Berg (1987b).

\section{Effect of trial length}

Trial length is an important factor in variability calculations. In this experiment we demonstrated that, as trial length increased, expressed as SD, increased as well, up to a certain maximum. Cycloversion variability reached a plateau after about $15-20 \mathrm{sec}$. Cyclovergence values were stable after 10-20 sec, depending on the subject. Previous investigators used shorter intervals for measuring torsion stability. Ferman et al. (1987a) used $4 \mathrm{sec}$ periods; Enright (1990) used periods of $5 \mathrm{sec}$, during which photographs were taken at $1 \mathrm{sec}$ intervals; Ott et al. (1992) used intervals that lasted $15 \mathrm{sec}$. SD values based on samples of such relatively short durations should be interpreted with care.

Because SD values had reached a plateau at the end of our $32 \mathrm{sec}$ measurements, we feel that these end-values properly represent the total variability.

\section{Perceptual demands}

Different noise levels for cyclovergence and cycloversion could reflect different perceptual demands. The most obvious function of cyclovergence is to promote retinal correspondence. The effect of cyclovergence errors on retinal correspondence is more pronounced in the peripheral retina, but there, receptive fields are larger as well. During the viewing of three-dimensional structures, retinal correspondence can never be complete and errors are larger in the peripheral visual fields. Although full correspondence is, thus, impossible, the oculomotor system may still play a role in its optimisation. Arguments for such optimisation have been presented by Van Rijn and Van den Berg (1993).

In theory, cyclovergence errors lead to misperception of (absolute) slant angles (Ogle \& Ellerbrock, 1946): a line that is slanted in the sagittal plane, viewed binocularly, gives rise to retinal images that are rotated in opposite directions in the left and right eye. Therefore, erros in cyclovergence could lead to misperception of slant. In contrast, cycloversion instability is expected to disturb the perception of tilt in the frontal plane.

Collewijn, Van der Steen and Van Rijn (1991) investigated thresholds for the perception of dynamic changes in tilt and slant of a single vertical line, oscillated at $0.25 \mathrm{~Hz}$. In the absence of any frame of reference the threshold for tilt perception was about $0.6 \mathrm{deg}$ and for slant perception about $2.4 \mathrm{deg}$. These values were measured as the threshold values for image cycloversion and cyclovergence, respectively, in the frontal plane, resulting from tilt and slant. They can, therefore, be directly related to ocular cycloversion and cyclovergence stability. Both threshold values are well above the insta- bility values for cycloversion and cyclovergence that we report here and, although we found that cyclovergence is more stable than cycloversion, thresholds for slant perception were highest. This seems to indicate that there is no direct relation between torsional stability of the eyes and the thresholds of either tilt or slant perception.

More indirect effects should, however, also be considered. For example, fluctuations of cyclovergence will induce changes in the horizontal disparity of targets above or below the plane of regard. Such changes will be opposite for targets in the upper and lower visual field, and may therefore disturb the estimation of relative depth of targets that are separated by some vertical distance. This is illustrated by the results of Enright (1990), who found that (static) equidistance estimates of two visual targets that were separated vertically were less accurate than equidistance estimates of targets that were separated horizontally. He demonstrated that the difference was accounted for by cyclovergence variability. $\mathrm{He}$ also showed that, when alternating fixation of the targets was allowed, estimates of both horizontally and vertically separated targets was far better and he argued that, therefore, cyclovergence instability does not affect slant percepion under natural conditions.

Recently, Ukwade, Bedell and White (1993) investigated patients with torsional congenital nystagmus. They found that tilt discrimination thresholds and, during foveation periods, variability values for torsion were in a similar range: tilt discrimination thresholds ranged from 0.2 to $1.4 \mathrm{deg}$ and torsion variability was about $0.6 \mathrm{deg}$ (SD). They found no differences between patients and controls. From their preliminary results they concluded that there is indeed a relation between tilt perception and variation of torsion.

\section{Cyclophoria}

The present experiment clearly demonstrates that in two of our subjects cyclophoria is present: in the absence of torsional visual cues, cyclovergence was systematically different from the situation in which cyclovergence could be controlled by visual feedback. Subject 1 had excyclophoria, i.e. in darkness the upper poles of both eyes rotated outward, while subject 2 had in-cyclophoria. It has been argued that a distinction between cyclotropia and cyclophoria is unjustified since a cyclodeviated eye does not correct itself when the other eye is covered (Von Noorden, 1985). We think that cyclophoria should not be judged on the basis of the position of one eye only, but on the basis of the relative position of the two eyes in absence and presence of (cyclo-) fusional stimuli. Of course this is only possible with techniques that allow measurement of eye positions in closed or covered eyes. This possibility is offered by the scleral coil technique. The other advantage of this technique is its sensitivity; the changes in cyclovergence amounting to about 2 deg in our subject 1 might easily remain unnoticed in clinical observation.

\section{Does the coil slip on the eye?}

Figure 4 shows that, over a whole session, there was a change in mean cyclovergence amounting to between 
2 and 4 deg (depending on the subject). This "long-term" change most likely reflects coil slippage in torsional direction. It is implausible that in our subjects there was a real build-up of cyclovergence during the session, particularly since intra-trial cyclovergence was very stable. It is also unlikely that a real "long-term" change could be due to a change in the position of one eye only (as was apparently the case in subjects 2 and 4). Finally, it is not likely that the systematic sequences of corrected and uncorrected cyclophoria, which we found in two subjects, were superimposed on real long-term cyclovergence changes. All these observations point in the direction of coil slippage as the cause. There was usually no relation between these "long-term" changes and within-trial trends (except for subject 4); therefore we think that this coil-slippage occurred mainly, if not solely, during inter-trial blinking periods. Notice that we instructed our subjects to blink quite vigorously between trials. Hence, we may assume that during blinking the coil tends to intort relative to the eye. This seems plausible, because the wire-leads from the coils are positioned in the nasal angle of the eye, and therefore the downward motion of the upper eye lid will exert an inward torque on the coil. This agrees with the long-term trend in all sessions.

In subject 4, intra-fixational trends, however small, were always directed oppositely to this long-term changes. In pilot experiments with combination coils we observed that after manual rotation of the coil on the eye, the coil sometimes tended to drift back to its original position. This indicates that coil-slippage has several components: (1) the coil may actually rotate on the surface of the conjunctiva; (2) rotation of the coil (e.g. by the eye lids) may cause some rotational drag of the conjunctiva on the underlying sclera, and this component may be restored by elastic forces when the external force subsides. This may be the reason for the systematic intra-trial drifts in subject 4 . We emphasize that in all subjects, including subject 4 , intra-trial trends were too small to account for any of the main effects (i.e. cycloversion vs cyclovergence stability and effects of background). This is further supported by the fact that coil slippage must affect cyclovergence more than cycloversion: (1) slip is unlikely to be conjugate in any case [compare Fig. 4(a, b)]; (2) the major trend of the slip was towards inward rotation in both eyes (Fig. 4); (3) cycloversion is calculated as the average torsion, therefore coil slippage of one eye appears at only half its size in cycloversion. For these reasons, if any intratrial slippage should have occurred, this would have decreased rather than increased the differences that we found between cyclovergence and cycloversion stability.

Photographic techniques do not have this problem of long-term slippage or drift. Enright (1990) reported inter-trial cyclovergence SD values of about $15 \mathrm{~min}$ arc, which was larger than the values for intra-trial stability, but far less than the values that would be expected on the basis of our $2-4$ deg drift.

\section{Role of head movements}

Movements of the head in torsional direction are compensated only partially by torsional eye movements since the gain of torsional VOR and torsional OKN is low: the combined torsional VOR and OKN has a gain of less than 0.7 (Collewijn et al., 1985; Seidman \& Leigh, 1989; see also Crawford \& Vilis, 1991; Van Rijn et al., $1992,1994)$. Since the effect of head roll is similar on both eyes, head movements may induce "artifactual" cycloversion with respect to the earth-fixed frame of reference, the field coils. Artifactual cyclovergence cannot be induced by head movements. The results from our control experiment (see Fig. 5) demonstrate that the contribution of roll head movements was very minor. In fact, head movements could ony account for about $5 \%$ of monocular eye torsion variability (calculated by comparing head variance to eye variance). This is far less than the differences that we found between cycloversion and cyclovergence stability. We may therefore conclude that these roll head movements cannot explain this difference. Thus, cycloversion instability was much larger than torsional head instability in our measurement conditions with the head supported. In contrast we found that in horizontal and vertical directions eye stability was better than head stability. This is in agreement with higher gains of VOR and OKN in these movement directions.

\section{CONCLUSIONS}

In this study we showed that spontaneous ocular torsion is largely conjugate. This implies that cyclovergence is controlled much better than cycloversion. We also showed that visual feedback enhances the stability of cyclovergence, but does not affect cycloversion stability. This cyclovergence/cycloversion difference was not secondary to roll head movements. We hypothesized that these differences reflect demands that are placed on optimisation of torsional retinal correspondence.

\section{REFERENCES}

Balliet, R. \& Nakayama, K. (1978), Training of voluntary torsion. Investigative Ophthalmology and Visual Science, 17, 303-314.

Carpenter, R. H. S. (1988). Movements of the eyes (2nd edn). London: Pion.

Collewijn, H., Van der Steen, J. \& Van Rijn, L. J. (1991). Binocular eye movements and depth perception. In Gorea, A., Fregnac, Y., Kapoula, Z. \& Findlay, J, Representations of vision. Trends and tacit assumptions in vision research (pp. 165-183). Cambridge: Cambridge University Press.

Collewijn, H., Van der Steen, J., Ferman, L. \& Jansen, T. C. (1985). Human ocular counterroll: Assessment of static and dynamic properties from electromagnetic scleral coil recordings. Experimental Brain Research, 59, 185-196.

Crawford, J. D. \& Vilis, T. (1991). Axes of eye rotation and Listing's law during rotations of the head. Journal of Neurophysiology, 65, $407-423$.

Crone, R. A. \& Everhard-Halm, Y. (1975). Optically induced eye torsion I. Fusional cyclovergence. Albrecht von Graefes Archiv für Klinische und Experimentelle Ophralmologie, 195, 231-239.

Diamond, S. G., Markham, C. H. \& Money, K. E. (1990). Instability of ocular torsion in zero gravity: Possible implications for space 
motion sickness. Aviation, Space and Environmental Medicine, 61, 899-905.

Enright, J. T. (1990). Stereopsis, cyclotorsional "noise" and the apparent vertical. Vision Research, 30, 1487-1497.

Erkelens, C. J. \& Collewijn, H. (1985). Eye movements and stereopsis during dichoptic viewing of moving random-dot stereograms. Vision Research, 25, 1689-1700.

Ferman, L., Collewijn, H. \& Van den Berg, A. V. (1987b). A direct test of Listing's law-II. Human ocular torsion measured under dynamic conditions. Vision Research, 27, 939-951.

Ferman, L., Collewijn, H., Jansen, T. C. \& Van den Berg, A. V. (1987a). Human gaze stability in the horizontal, vertical and torsional direction during voluntary head movements, evaluated with a three-dimensional scleral induction coil technique. Vision Research, 27, 811-828.

Glanz, S. A. (1987). A primer of biostatistics (2nd edn). New York: McGraw-Hill.

Haustein, W. (1989). Considerations on Listing's law and the primary position by means of a matrix description of eye position control. Biological Cybernetics, 60, 411-420.

Howard, I. P. \& Zacher, J. E. (1991). Human cyclovergence as a function of stimulus frequency and amplitude. Experimental Brain Research, 85, 445-450.

Julesz, B. (1965). Texture and visual perception. Scientific American, 212(2), 38-48.

Kertesz, A. E. (1983). Vertical and cyclofusional disparity vergence. In Schor, C. M. \& Ciuffreda, K. J. (Eds), Vergence eye movements: Basic and clinical aspects (pp. 317-348). London: Butterworths.

Ogle, K. N. \& Ellerbrock, V. J. (1946). Cyclofusional movements. Archives of Ophthalmology, 36, 700-735.

Ott, D., Seidman, S. H. \& Leigh, R. J. (1992). The stability of human eye orientation during visual fixation. Neuroscience Letters, 142, 183-186.

Robinson, D. A. (1963). A method of measuring eye movement using a scleral search coil in a magnetic field. IEEE Transactions on Biomedical Electronics, BME-10, 137-145.

Seidman, S. H. \& Leigh, R. J. (1989). The human torsional vestibulo- ocular reflex during rotation about an earth-vertical axis. Brain Research, 504, 264-268.

Skavenski, A. A., Hansen, R. M., Steinman, R. M. \& Winterson, B. J. (1979). Quality of retinal image stabilization during small natural and artificial body rotations in man. Vision Research, 19, 675-683.

Steinman, R. M. (1986). Eye movement. Vision Research, 26, $1389-1400$.

Steinman, R. M. \& Collewijn, H. (1980). Binocular retinal image motion during active head rotation. Vision Research, 20, 415-429.

Steinman, R. M., Levinson, J. Z., Collewijn, H. \& Van der Steen, J. (1985). Vision in the presence of known natural retinal image motion. Journal of the Optical Society of America, A, 226-233.

Tweed, D., Cadera, W. \& Vilis, T. (1990). Computing three-dimensional eye position quaternions and eye velocity from search coil signals. Vision Research, 30, 97-110.

Ukwade, M. T., Bedell, H. E. \& White, J. M. (1993). Orientation discrimination and variability of torsional eye position in congenital nystagmus. Investigative Ophthalmology and Visual Science (Supplement), 34, 1125.

Van Rijn, L. J. \& Van den Berg, A. V. (1993). Binocular eye orientation during fixations: Listing's law extended to include eye vergence. Vision Research, 33, 691-708.

Van Rijn, L. J. \& Van der Steen, H. (1992). Stability of the human eyes in torsional direction: Short term spontaneous torsional eye movements are conjugate. Pflügers Archiv (Supplement), 420, R42.

Van Rijn, L. J., Van der Steen, J. \& Collewijn, H. (1992). Visually induced cycloversion and cyclovergence. Vision Research, 32, $1875-1883$.

Van Rijn, L. J., Van der Steen, J. \& Collewijn, H. (1994). Eye torsion elicited by oscillating gratings: Effects of orientation, wavelength and stationary contours. Vision Research, 34, 533-540.

Von Noorden, G. K. (1985). Burian-Von Noorden's Binocular vision and ocular motility, theory and management of strabismus (3rd edn). St Louis: Mosby.

Westheimer, G. \& McKee, S. P. (1975). Visual acuity in the presence of retinal-image motion. Journal of the Optical Society of America, $65,847-850$. 